\title{
Design of Vehicle Antenna Servo Tracking System Controller
}

\author{
FAN Qiang ${ }^{1, a}$, WANG $\mathrm{Yu}^{2, \mathrm{~b}}$ \\ ${ }^{1}$ School of Automation, Beijing Institute of Technology, Beijing, 100081, China \\ ${ }^{2}$ School of Automation, Beijing Institute of Technology, Beijing, 100081, China \\ a15210622615@163.com, bwangyu@bit.edu.cn
}

Keywords: Mobile Satellite Communication; NPID controller; feed-forward control

\begin{abstract}
Mobile satellite communication system is required to maintain stable satellite communications in the vehicle movement. Because of the different road conditions, all kinds of postures information of satellite antenna has the characteristics which are complex and fast changing. This paper presents an azimuth servo controller based on nonlinear PID (referred to as "NPID"), and such controller has strong ability of anti-load speed disturbance. Through MATLAB simulation and comparing the time-domain characteristics of NPID and classic PID controller, it can be verified that NPID outperforms PID control. Especially in the situation of fast cornering and the speed can be measured, the NPID controller with feed-forward is superior to the classic PID controller with feed-forward in terms of performance of vehicle disturbance suppression.
\end{abstract}

\section{Introduction}

Mobile satellite communication system is required to keep stable and reliable satellite communications while the vehicle is moving. Because of the different road conditions, all kinds of satellite antenna attitude information will change at any time, the servo system requires fast response speed to be able to shield the disturbance from the outside world [1]. This research adopts flat satellite antenna, which receives signal with narrower beam angle. In order to ensure the high quality of satellite communication, it must improve the control precision and speed of the servo control system. At present, mobile satellite communication system mainly uses the simple classical PID control strategy. However, due to the PID algorithm is based on the error feedback to eliminate the error, and by increasing the proportional gain to improve the rapidity of system while a large proportional gain is easy to cause the system to overshoot, it is not conducive to the satellite signal tracking. Meanwhile the PID controller is sensitive to the gain of PID, so its adaptability to changing situations is poor. In addition, when there is a large vehicle disturbance in the system, it is difficult to meet the control demand by using the classic PID control algorithm to suppress, because the inhibitory effect is limited [2]. In contrast, the nonlinear PID (NPID) controller has better inhibitory ability when the parameters and structure of object changes, and this algorithm is simple and easy to implement. In order to solve the contradiction between the overshoot and rapidity, Pre-arranged transition process by tracking differentiator is a very effective way. The design of this NPID controller based on tracking differentiator (TD) and nonlinear states error feedback control laws (NLSEF), combining the feed forward control technology, which applies to the satellite antenna servo system. When the system load has a sudden strong disturbance, the controller has strong anti-interference ability.

\section{Azimuth servo mathematical model}

The antenna azimuth servo control system mainly consists of DC servo motor, motor drive, speed reducer and azimuth turntable. The mathematical model is created based on the motor speed loop as shown in Fig. 1 [3]. 


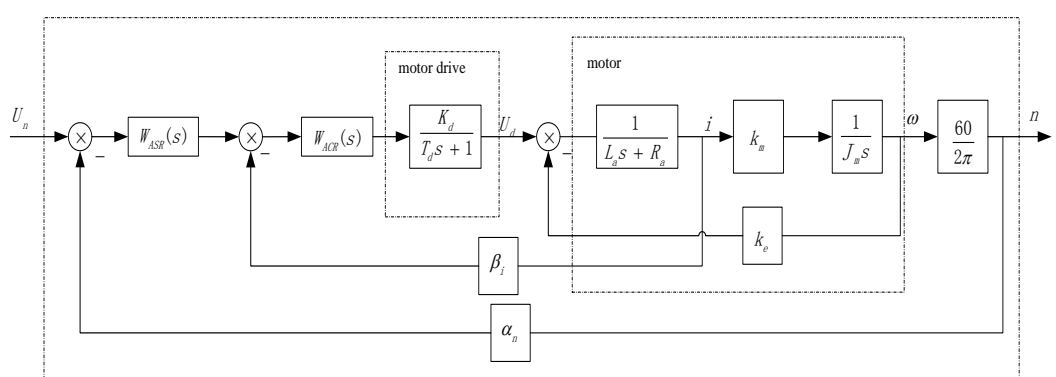

Fig. 1: Mathematical model structure based on the motor speed loop

Wherein the transfer function of the motor is:

$G(s)=\frac{\omega(s)}{u_{d}(s)}=\frac{k_{m}}{\left(L_{a} s+R_{a}\right) J_{m} s+k_{e} k_{m}}$

When $L_{a}$ is small, the motor transfer function can be simplified to:

$G(s)=\frac{k_{m}}{R_{a} J_{m} s+k_{e} k_{m}}=\frac{K}{T_{m} s+1}$

Wherein $T_{m}$ is motor mechanical constant:

$T_{m}=\frac{R_{a} J_{m}}{k_{e} k_{m}}, K=\frac{1}{k_{e}}$.

Motor drive model can be simplified to a first order inertia link:

$G_{d}(s)=\frac{K_{d}}{T_{d} s+1}$

Wherein the parameter $K_{d}$ and $T_{d}$ are obtained by the actual system step response curve fitting. The motor related parameters are brought into type (2), (3), we get the mathematical model based on the drive and motor series.

$$
\frac{n(s)}{u_{n}(s)}=\frac{892.1691}{s^{2}+39.427344 s+892.1691}
$$

Type (4) is the mathematical model that is established based on the motor speed loop, namely input and output are based on RPM as a unit. Combining with the speed reducer (reduction ratio is 1:114) and azimuth turntable can obtain the structure diagram of the controlled object model as shown in Fig. 2.

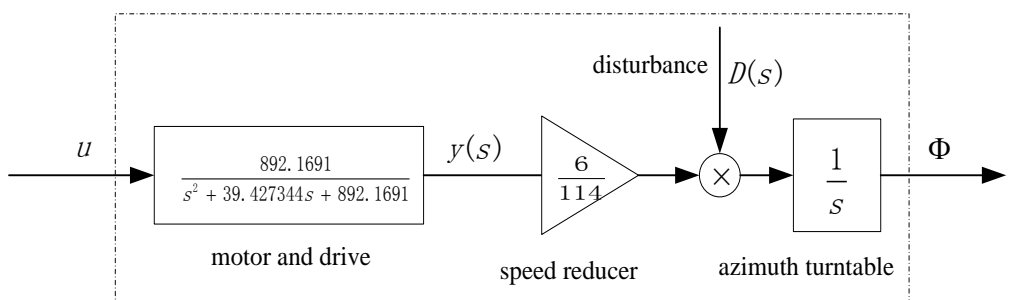

Fig.2: The structure of the controlled object model

\section{The design of NPID controller}

Due to the working environment of vehicle antenna control system is very complex, there is a variety of outside interference that affects the system tracking precision, at the same time, although the motor and drive model of the control system has been got, but this is only an approximation model, which has a certain gap with the actual system. Therefore, in order to guarantee the stability and tracking accuracy of the system, this paper explores the use of nonlinear PID control algorithm to control the azimuth servo system.

The NPID controller is composed of two parts, which are tracking differentiator (TD) and nonlinear states error feedback control laws (NLSEF). 
In order to solve the contradiction between the overshoot and rapidity, Pre-arranged transition process by tracking differentiator is a very effective way. It can make the choice of the error feedback gain and each order error differential feedback gain to be expanded, making it easier tuning. And it can make the given feedback gain can adapt to object parameter range extension, namely the robust of the controller is more strengthen. This design uses the steepest discrete tracking differentiator to arrange transition process.

$$
\left\{\begin{array}{l}
f h=f h a n\left(v_{1}(k)-v(k), v_{2}(k), r_{1}, h_{1}\right) \\
v_{1}(k+1)=v_{1}(k)+h v_{2}(k) \\
v_{2}(k+1)=v_{2}(k)+h f h
\end{array}\right.
$$

Wherein $V$ is a given input signal, fhan $(\sqcup)$ is the speed control integrated function[4], $V_{1}$ is angle tracking signal , $V_{2}$ is first derivative signal,$h$ is the sampling step , $r_{1}$ is fast factor,$h_{1}$ is the filtering factor , usually $h_{1}=n * h(n>=1)$. The parameters $r_{1}$ and $h_{1}$ are required for commissioning.

Using the nonlinear TD can obtain transition process $V_{1}$ and the corresponding derivative $V_{2}$ of the reference input $V$. And through navigation attitude system of mobile satellite communication system, we can obtain the turntable angle $\Phi$ and speed variables $\omega$. We can complete the design of the following NLSEF using these two groups of variables.

$$
\left\{\begin{array}{l}
e_{1}=v_{1}-\Phi, e_{2}=v_{2}-\omega \\
u_{0}=- \text { fhan }\left(e_{1}, c e_{2}, r_{2}, h_{2}\right)+k v_{2}
\end{array}\right.
$$

In the formula, fhan $(\sqcup)$ can be used to nonlinear state feedback configuration which has rapidity and eliminate flutter of special functions, and the parameter $c$ is damping term [5]. What deserves special mention is that using the extracted first order differential signal of the transition process as NPID feed-forward control can improve the rapidity of the system. The parameters $r_{2}, h_{2}, c$ and $k$ of the NLSEF are required for commissioning.

In order to overcome the large vehicle disturbance, combining the NPID with PD feed-forward control technology is used to control. This PD feed-forward controller is established based on measurable disturbance signal. And Fig. 3 is the azimuth servo system control diagram.

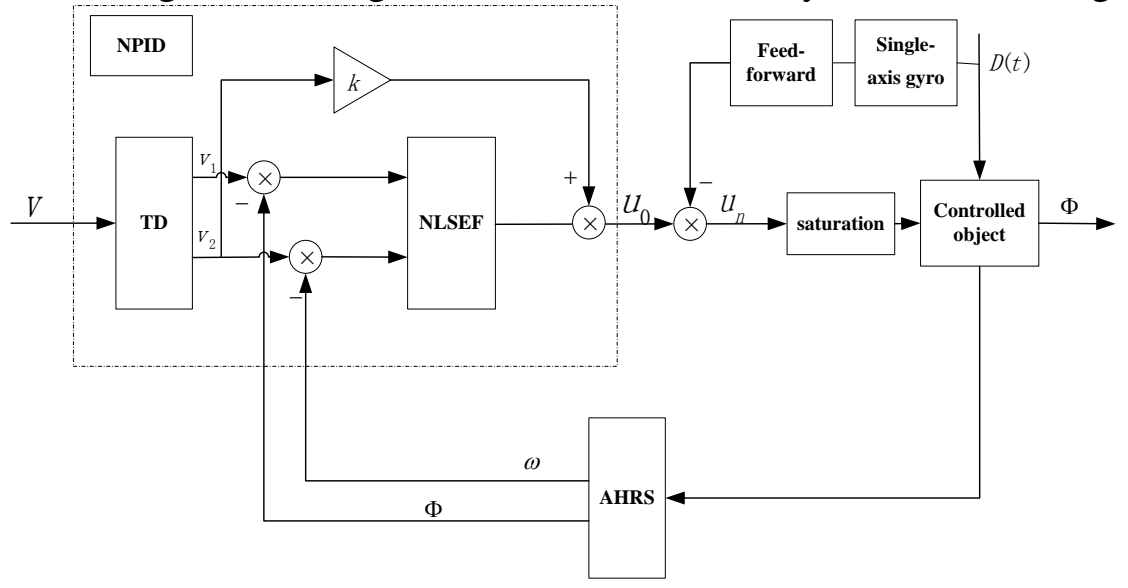

Fig.3: The azimuth servo system control diagram

\section{System simulation}

In order to verify the control performance of the designed NPID, We through the following two aspects to carry on the MATLAB simulation, compared control effect of it with classical PID. 1) The step response; 2) Research on angular deviation of antenna aims at satellite in the vehicle fast cornering.

We completed this NPID parameters debugging through trial and error method. The sampling step length of discrete system $h=0.01$, TD section: $r_{1}=100, h_{1}=0.02$; NLSEF section: $r_{2}=3000, h_{2}=0.05$, $c=1.5, k=17$. To meet the rapidity of system, the classic PID debugging parameters are $k_{p}=175, k_{i}=0$, $k_{d}=1.25$. The PD feed-forward control parameters of NPID and PID controller both are $k_{p}{ }^{\prime}=1$, $k_{d}{ }^{\prime}=0.045$. 
Applying a unit step function to the system, namely $v(t)=1(t>0)$, Unit: degree. Fig. 4 is the comparison diagram between the transition process of set point and the step response of NPID controller. Fig. 5 is the step response of NPID and PID comparison diagram.

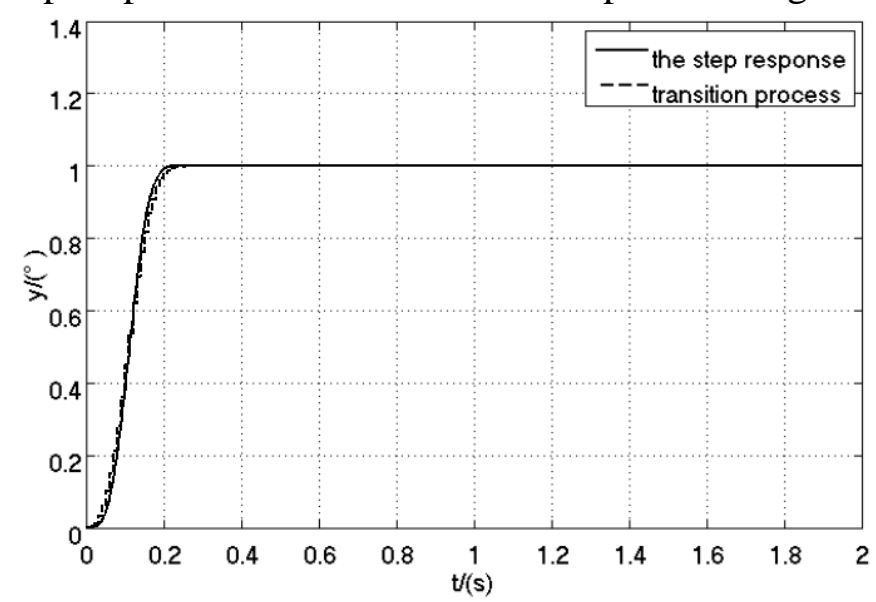

Fig. 4: The transition process and the step response of NPID

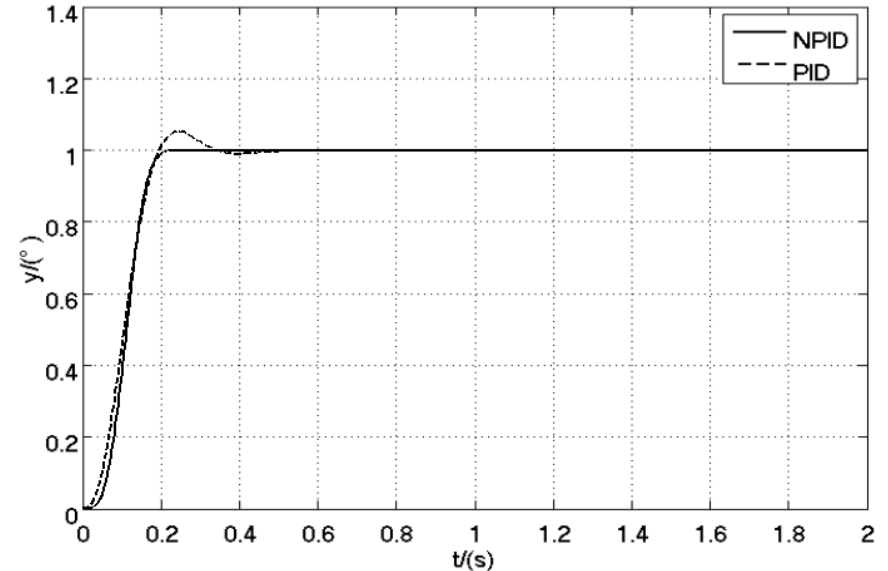

Fig. 5: The step response of NPID and PID

From the simulation results in Fig. 4, we can know that the solid line is the step response curve of NPID, the dotted line is the set point transition process of NPID, and the two curves substantially coincide. Due to the transition process without overshoot, and through tuning parameters to meet the system requirement of rapidity, just below the system's capacity, combined with nonlinear combination, we can achieve fast response with no overshoot results.

From the simulation results in Fig. 5, we can know that the solid line is the control result of NPID, and the dotted line is the control result of PID. As can be seen from the figure, while required to meet the fast condition, NPID adjustable curve is smooth and without overshoot, but the PID regulating curve has $5 \%$ overshoot. So we can see that the time domain characteristic of designed NPID is superior to classical PID.

Because of the high accuracy requirement of the antenna azimuth angle, when the deviation is greater than $0.2^{\circ}$ will cause the signal attenuation that lost satellite quickly, therefore, making the antenna still aim at the satellite in the vehicle fast cornering is the most critical problem. Because the vehicle speed can be measured by single-axis gyro, we combine with feed-forward control technique to control the system.

$$
D_{1}(t)=\left\{\begin{array}{l}
200 t(0<t \leq 0.75) \\
150(0.75<t \leq 3) \\
150-200(t-3)(3<t \leq 3.75) \\
0(t>3.75)
\end{array},(\text { degree } / \mathrm{sec}) ; D_{2}(t)=150 \sin (2 t),(\text { degree } / \mathrm{sec}) .\right.
$$

These two given functions are as the speed of vehicle continuously cornering. Figs 6, 7 respectively are the response comparison diagrams of NPID and PID controller with feed-forward under the effect of the disturbances $D_{1}, D_{2}$. 


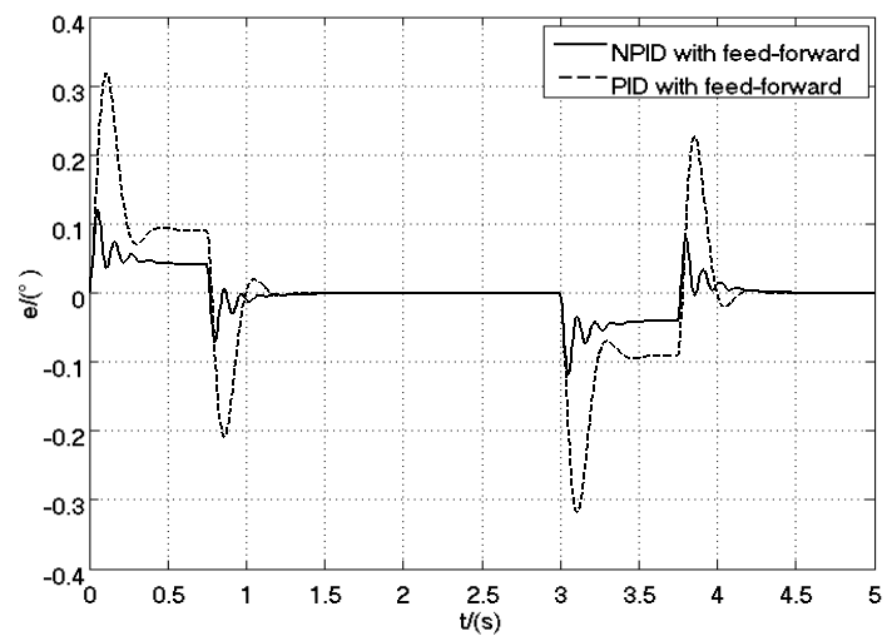

Fig. 6: Under the effect of disturbance $D_{1}$ angular deviation of antenna aims at satellite comparison diagram

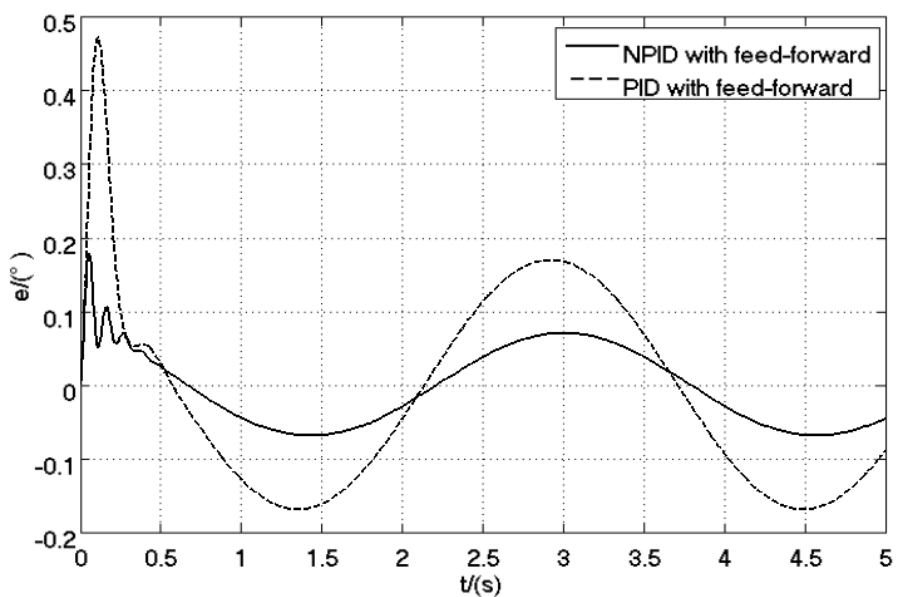

Fig.7: Under the effect of disturbance $D_{2}$ angular deviation of antenna aims at satellite comparison diagram

Figs 6, 7 show that the angular deviation of antenna aims at satellite is largest at the initial moment, under the effect of disturbance $D_{1}$, PID maximum deviation $e=0.32^{\circ}$ and NPID maximum deviation $e=0.12^{\circ}$; under the effect of disturbance $D_{2}$, PID maximum deviation $e=0.47^{\circ}$ and NPID maximum deviation $e=0.18^{\circ}$, this is because there is a great instantaneous jerk at the initial time. Under the effect of the same disturbance, the maximum deviation of NPID is significantly less than PID. At any other time, the ability of NPID to inhibit the disturbance also is better than PID control. This is because the classic PID has a fixed gain, the control is stronger when the deviation is larger, and the control is weaker when the deviation is smaller, but the NPID selects nonlinear function which has the characteristics of small deviation big gain, small gain of large deviation. When the vehicle disturbance exists, and the angular deviation is small, the NPID controller with feed-forward has a strong ability to eliminate deviation. In short, because of anti-interference performance improved significantly, the angular deviation of antenna aims at satellite can be controlled within the required angle, so as to meet the demand.

\section{Conclusion}

In this paper, we have studied the antenna azimuth control problems in the mobile satellite communication system and designed a kind of NPID controller with feed-forward. Through the simulation, compared with the classic PID control algorithm, NPID has better rapidity and stability on the azimuth control; and it has a better ability to reduce the angular deviation in the vehicle fast cornering. In future work, we will continue to optimize the parameters of this NPID, and take this algorithm to apply to the actual system. 


\section{References}

[1] Tao Yu, Zhu Jun. Research on Tracking Beam Pointing Error for Multi-planar Antenna SOTM [J]. China Cable Television. 2012(2):137-139.

[2] Shang Hong, Chen Zhi-min, Ren Yong-ping. Parameter selection for a new class of nonlinear PID controller [J]. Control Theory \& Applications. 2009, 26(4):439-442.

[3] Liao Xiao-zhong, Liu Xiang-dong. Automatic control system [M].Beijing: Beijing Institute of Technology Press, 2011:66.

[4] Han Jing-qing, Wang Wei. Nonlinear tracking-differentiator [J]. Systems Science and Mathematical Sciences. 1994, 14(2):177-183.

[5] Han Jing-qing. Active Disturbance Rejection Control Technique-the technique for estimating and compensating the uncertainties [M].Beijing: National Defense Industry Press, 2013:144-146. 